PROCEEDINGS OF THE

AMERICAN MATHEMATICAL SOCIETY

Volume 132, Number 3, Pages 769-780

S 0002-9939(03)07096-5

Article electronically published on July 29, 2003

\title{
NEVANLINNA-PICK INTERPOLATION: PICK MATRICES HAVE BOUNDED NUMBER OF NEGATIVE EIGENVALUES
}

\author{
V. BOLOTNIKOV, A. KHEIFETS, AND L. RODMAN
}

(Communicated by Joseph A. Ball)

\begin{abstract}
The Nevanlinna-Pick interpolation problem is studied in the class of functions defined on the unit disk without a discrete set, with the property that all their Pick matrices have not more than a prescribed number of negative eigenvalues. It is shown, in particular, that the degenerate problem always has a unique solution, not necessarily meromorphic. A related extension problem to a maximal function in the class is also studied.
\end{abstract}

\section{INTRODUCTION}

Let $\mathbb{D}$ be the open unit disk. We say that a set $\Lambda \subset \mathbb{D}$ is discrete (in $\mathbb{D}$ ) if $\Lambda$ is at most countable, with accumulation points (if any) only on the boundary of $\mathbb{D}$. Throughout the paper, Dom $(f)$ denotes the domain of definition of a function $f$.

Definition 1.1. Given a nonnegative integer $\kappa$, the class $\mathcal{S}_{\kappa}$ consists of (complexvalued) functions $f$ defined on $\operatorname{Dom}(f)=\mathbb{D} \backslash \Lambda$, where $\Lambda=\Lambda(f)$ is a discrete set, and such that all Pick matrices (which are clearly Hermitian)

$$
P_{n}\left(f ; z_{1}, \ldots, z_{n}\right):=\left[\frac{1-f\left(z_{i}\right) f\left(z_{j}\right)^{*}}{1-z_{i} z_{j}^{*}}\right]_{j, i=1}^{n}, \quad z_{1}, \ldots, z_{n} \in \mathbb{D} \backslash \Lambda,
$$

have at most $\kappa$ negative eigenvalues (counted with multiplicities), and at least one such Pick matrix has exactly $\kappa$ negative eigenvalues.

It is well known that every function $f$ in $\mathcal{S}_{0}$ admits an extension to a $S c h u r$ function, i.e., a function that is analytic in $\mathbb{D}$ and maps $\mathbb{D}$ into the closed unit disk. Here and elsewhere, we say that a function $g$ is an extension of a function $f$ if $\operatorname{Dom}(g) \supseteq \operatorname{Dom}(f)$ and $g(z)=f(z)$ for every $z \in \operatorname{Dom}(f)$. Slightly abusing notation, in the sequel we use $\mathcal{S}_{0}$ to denote the class of Schur functions.

Meromorphic functions in the class $\mathcal{S}_{\kappa}$ have been studied before in various contexts: spectral theory of unitary operators in Pontryagin spaces 14, model theory [5], and factorizations [14. Interpolation has been considered in the class of meromorphic functions in $\mathcal{S}_{\kappa}$ : Schur-Takagi problem [1], Nevanlinna-Pick problem [17], 13, 8. However, not all functions in $\mathcal{S}_{\kappa}$ are meromorphic. Non-meromorphic functions in $\mathcal{S}_{\kappa}$ appear as almost multipliers (or pseudomultipliers) of the Hardy

Received by the editors September 12, 2002 and, in revised form, October 23, 2002.

2000 Mathematics Subject Classification. Primary 41A05, 32A35.

Key words and phrases. Pick matrices, negative squares, Nevanlinna-Pick interpolation.

The research of the third author was supported in part by NSF grant DMS-9988579. 
$H_{2}$ space; see 4 for a general theory of pseudomultipliers in reproducing kernel Hilbert spaces. Connections between the pseudomultiplier theory and the structure of functions in the class $\mathcal{S}_{\kappa}$ (see, in particular, Theorem 1.3 below) were discussed in detail in [10].

In what follows, we denote by $Z(f)$ the zero set for $f: Z(f)=\{z \in \operatorname{Dom}(f)$ : $f(z)=0\}$. Recall that a (finite) Blaschke product is a rational function $B(z)$ that is analytic on $\mathbb{D}$ and unimodular on the unit circle $\mathbb{T}:|B(z)|=1$ for $|z|=1$; the degree of $B(z)$ is the number of zeros (counted with multiplicities) of $B(z)$ in $\mathbb{D}$.

Definition 1.2. A function $f$ is said to be a standard function if it has the form

$$
f(z)=\left\{\begin{array}{ccc}
\frac{S(z)}{B(z)} & \text { if } & z \notin \mathcal{W} \cup \mathcal{Z} \\
\gamma_{j} & \text { if } & z=z_{j} \in \mathcal{Z}
\end{array}\right.
$$

for some complex numbers $\gamma_{1}, \ldots, \gamma_{\ell}$, where:

(1) $\mathcal{Z}=\left\{z_{1}, \ldots, z_{\ell}\right\}$ and $\mathcal{W}$ are disjoint finite sets in $\mathbb{D}$;

(2) $B(z)$ is a Blaschke product of degree $q \geq 0$ and $S(z)$ is a Schur function with the zero sets $Z(B)$ and $Z(S)$, respectively, such that

$$
\mathcal{W} \subseteq Z(B) \subseteq \mathcal{W} \cup \mathcal{Z} \quad \text { and } \quad Z(B) \cap Z(S)=\emptyset
$$

(3) if $z_{j} \in \mathcal{Z} \backslash Z(B)$, then $\frac{S\left(z_{j}\right)}{B\left(z_{j}\right)} \neq \gamma_{j}$.

For the standard function $f$ of the form (1.2), Dom $(f)=\mathbb{D} \backslash \mathcal{W}$. The meromorphic function $S(z) / B(z)$ will be called the meromorphic part of $f$. More informally, $\mathcal{Z}$ is the set of points $z_{0}$ at which $f$ is defined and $f\left(z_{0}\right) \neq \lim _{z \rightarrow z_{0}} f(z)$. The case when $\lim _{z \rightarrow z_{0}}|f(z)|=\infty$ is not excluded here; in this case $f\left(z_{0}\right)$ is defined nevertheless. The set $\mathcal{W}$ consists of those poles of $S(z) / B(z)$ at which $f$ is not defined. In reference to the properties (1)-(3) in Definition [1.2 we will say that $f(z)$ has $q$ poles (the zeros of $B(z)$ ), where each pole is counted according to its multiplicity as a zero of $B(z)$, and $\ell$ jumps $z_{1}, \ldots, z_{\ell}$. Note that the poles and jumps need not be disjoint.

Theorem 1.3. Let $f$ be defined on $\mathbb{D} \backslash \Lambda$, where $\Lambda$ is a discrete set. Then $f$ belongs to $\mathcal{S}_{\kappa}$ if and only if $f$ admits an extension to a standard function with $\ell$ jumps (for some $\ell \leq \kappa)$ and $\kappa-\ell$ poles, and the jumps of the standard function are contained in $\mathbb{D} \backslash \Lambda$.

Theorem 1.3 was proved in [4], and also in [10] using a different approach than that found in 4 .

In this paper we consider the Nevanlinna-Pick interpolation problem for (not necessarily meromorphic) functions $F \in \mathcal{S}_{\kappa}$ :

$\mathbf{N P}_{\kappa}:$ Given points $z_{1}, \ldots, z_{k} \in \mathbb{D}$ and complex numbers $F_{1}, \ldots, F_{k}$, find all functions $F \in \mathcal{S}_{\kappa}$ such that $z_{1}, \ldots, z_{k}$ belong to the domain of definition of $F$ and

$$
F\left(z_{i}\right)=F_{i} \quad(i=1, \ldots, k) .
$$

If $F$ is a solution of the $\mathbf{N P}_{\kappa}$ problem, then the Pick matrix $P_{k}\left(F ; z_{1}, \ldots, z_{k}\right)$ takes the form

$$
P=\left[\frac{1-F_{i} F_{j}^{*}}{1-z_{i} z_{j}^{*}}\right]_{i, j=1}^{k} .
$$


This matrix is the same for every solution $F$ of the problem and depends only on the interpolation data. It is called the Pick matrix of the $\mathbf{N P}_{\kappa}$ problem. It is easily seen that $P$ is a unique solution to the Stein equation

$$
P-T P T^{*}=E E^{*}-C C^{*},
$$

where

$$
T=\left[\begin{array}{ccc}
z_{1} & & \\
& \ddots & \\
& & z_{k}
\end{array}\right], \quad E=\left[\begin{array}{c}
1 \\
\vdots \\
1
\end{array}\right], \quad C=\left[\begin{array}{c}
F_{1} \\
\vdots \\
F_{k}
\end{array}\right] .
$$

It is clear that if $F \in \mathcal{S}_{\kappa}$ satisfies conditions (1.3), then sq- $_{-}(P) \leq \kappa$, which is therefore a necessary condition for the $\mathbf{N P}_{\kappa}$ problem to have a solution. (Here and elsewhere we denote by $\mathrm{sq}_{-}(X)$ the number of negative eigenvalues, counted with multiplicities, of a Hermitian matrix $X$.) On the other hand, if

$$
\text { sq_}_{-}(P)=\kappa \text { and } \operatorname{det} P \neq 0 \text {, }
$$

then the $\mathbf{N P}_{\kappa}$ problem has infinitely many solutions, which are parametrized by a linear fractional transformation. Let $\Theta(z)$ be the $\mathbb{C}^{2 \times 2}$-valued function defined by

$$
\begin{aligned}
\Theta(z) & =\left[\begin{array}{ll}
\Theta_{11}(z) & \Theta_{12}(z) \\
\Theta_{21}(z) & \Theta_{22}(z)
\end{array}\right] \\
& =I_{2}+(z-1)\left[\begin{array}{l}
E^{*} \\
C^{*}
\end{array}\right]\left(I-z T^{*}\right)^{-1} P^{-1}(I-T)^{-1}\left[\begin{array}{ll}
E & -C
\end{array}\right] .
\end{aligned}
$$

It turns out that $\Theta$ is $J$-unitary on $\mathbb{T}$ with respect to the signature matrix

$$
J=\left[\begin{array}{cc}
1 & 0 \\
0 & -1
\end{array}\right],
$$

i.e., $\Theta(z) J \Theta(z)^{*}=J$ for $z \in \mathbb{T}$. Using (1.8), we have

$$
\begin{aligned}
J-\Theta(z) J \Theta(w)^{*}= & {\left[\begin{array}{l}
E^{*} \\
C^{*}
\end{array}\right]\left(I-z T^{*}\right)^{-1} P^{-1}(I-T)^{-1} } \\
& \times L(z, w)\left(I-T^{*}\right)^{-1} P^{-1}\left(I-w^{*} T\right)^{-1}\left[\begin{array}{ll}
E & C
\end{array}\right],
\end{aligned}
$$

where

$$
\begin{aligned}
L(z, w)= & (1-z)\left(I-w^{*} T\right) P\left(I-T^{*}\right)+\left(1-w^{*}\right)(I-T) P\left(I-z T^{*}\right) \\
& -(1-z)\left(1-w^{*}\right)\left(E E^{*}-C C^{*}\right) \\
= & \left(1-z w^{*}\right)(I-T) P\left(I-T^{*}\right) \quad(\text { using }(1.5)) ;
\end{aligned}
$$

hence

$$
J-\Theta(z) J \Theta(w)^{*}=\left(1-z w^{*}\right)\left[\begin{array}{l}
E^{*} \\
C^{*}
\end{array}\right]\left(I-z T^{*}\right)^{-1} P^{-1}\left(I-w^{*} T\right)^{-1}\left[\begin{array}{ll}
E & C
\end{array}\right]
$$

holds for every choice of points $z$ and $w$ at which $\Theta$ is analytic. (The above calculation can be found in many sources, in various forms.) We note also that

$$
\operatorname{det} \Theta(z)=\prod_{j=1}^{k} \frac{\left(z-z_{j}\right)\left(1-z_{j}^{*}\right)}{\left(1-z z_{j}^{*}\right)\left(1-z_{j}\right)}
$$

and thus $\Theta(z)$ is invertible at each point $z \in \mathbb{D} \backslash\left\{z_{1}, \ldots, z_{k}\right\}$. 
The following theorem (for the proof see [6, Section 19.3]) gives a complete description of all meromorphic functions $F \in \mathcal{S}_{\kappa}$ that are analytic at $z_{1}, \ldots, z_{k}$ and satisfy interpolation conditions (1.3).

Theorem 1.4. Let the Pick matrix $P$ of the $\mathbf{N P}_{\kappa}$ problem meet conditions (1.7) and let $\Theta$ be the function given in (1.8). Then all meromorphic solutions $F$ of the $\mathbf{N P}_{\kappa}$ problem are parametrized by the fractional linear transformation

$$
F(z)=\mathbf{T}_{\Theta}[\mathcal{E}]:=\frac{\Theta_{11}(z) \mathcal{E}(z)+\Theta_{12}(z)}{\Theta_{21}(z) \mathcal{E}(z)+\Theta_{22}(z)}
$$

where the parameter $\mathcal{E}$ runs through the set of those Schur functions that satisfy

$$
\Theta_{21}\left(z_{i}\right) \mathcal{E}\left(z_{i}\right)+\Theta_{22}\left(z_{i}\right) \neq 0 \quad(i=1, \ldots, k) .
$$

It can be shown that if $P$ is not singular, there are infinitely many Schur functions $\mathcal{E}$ satisfying (1.12) and therefore the $\mathbf{N P}_{\kappa}$ problem indeed has infinitely many solutions.

The solution of the $\mathbf{N P}_{\kappa}$ problem in the nondegenerate case (when (1.7) is satisfied) is given by the following theorem, which is one of the main results of this paper. In view of Theorem 1.3. we need only to describe the standard solutions of $\mathbf{N P}{ }_{\kappa}$, i.e., those solutions that are standard functions in the sense of Definition 1.2

Theorem 1.5. Assume that the Pick matrix $P$ of the $\mathbf{N P}_{\kappa}$ problem satisfies (1.7), and let $\Theta$ be the function given in (1.8). Then all standard solutions $F$ of the $\mathbf{N P}_{\kappa}$ problem are parametrized by the formula

$$
F(z)=\left\{\begin{array}{cl}
\frac{\Theta_{11}(z) \mathcal{E}(z)+\Theta_{12}(z)}{\Theta_{21}(z) \mathcal{E}(z)+\Theta_{22}(z)} & \text { if } z \notin\left(\left\{z_{1}, \ldots, z_{k}\right\} \cup\left\{\text { zeros of } \Theta_{21} \mathcal{E}+\Theta_{22}\right\}\right) \\
F_{i} & \text { if } z=z_{i}(i=1, \ldots, k)
\end{array}\right.
$$

where the parameter $\mathcal{E}$ runs through the class of Schur functions $\mathcal{S}_{0}$.

Note that conditions (1.12) are absent in Theorem 1.5 The difference between Theorems 1.4 and 1.5 is that in Theorem 1.5 we consider all parameters $\mathcal{E} \in \mathcal{S}_{0}$ including those that do not satisfy at least one of inequalities (1.12). In the context of meromorphic interpolation, these parameters are called excluded [13], [12], since they do not lead, via (1.11), to meromorphic solutions of $\mathbf{N} \mathbf{P}_{\kappa}$.

A problem similar (but not equivalent) to $\mathbf{N P}_{\kappa}$, namely, the following Sarason formulation of the interpolation conditions, was studied in [7] using the negative squares version of commutant lifting: Given $h \in H^{\infty}$ that satisfies $h\left(z_{i}\right)=F_{i}$, $i=1, \ldots, k$, and an inner function $\theta$ (which we take to be the finite Blaschke product with zeros at the points $z_{1}, \ldots, z_{k}$ ), describe functions $f$ with the properties that the multiplication operator $M_{f}$ maps $B H^{2}$ into $H^{2}$ for some Blaschke product $B$ of degree $\kappa$ such that $f B=(h+\theta q) B$ for some $q \in H^{\infty}$ and $\left\|M_{f}\right\| \leq 1$.

The proof of Theorem 1.5] is given in the next section. In Section 3 we study the degenerate $\mathbf{N P}_{\kappa}$ problem, i.e., such that the pick matrix is singular, and prove that the solution is unique. As an application of the results on the $\mathbf{N P}_{\kappa}$ problem, we prove in Section 4 that every function in the class $\mathcal{S}_{\kappa}$ relative to its domain of definition can be extended to a standard function in $\mathcal{S}_{\kappa}$. In the same section we give an example showing that this statement is generally false if only meromorphic functions in $\mathcal{S}_{\kappa}$ are considered. 


\section{Proof of Theorem 1.5}

We need some results from [9, which will now be recalled. In what follows, we use the notation

$$
U_{\mathcal{E}}(z)=\Theta_{11}(z) \mathcal{E}(z)+\Theta_{12}(z), \quad V_{\mathcal{E}}(z)=\Theta_{21}(z) \mathcal{E}(z)+\Theta_{22}(z) .
$$

A Schur function $\mathcal{E}$ is said to be a parameter of multi-order $\mathbf{m}=\left(m_{1}, \ldots, m_{k}\right)$ of the transformation (1.11) if the function $V_{\mathcal{E}}=\Theta_{21} \mathcal{E}+\Theta_{22}$ has zeros of multiplicities $m_{i}$ at $z_{i}$, respectively, for $i=1, \ldots, k$. Here the $m_{i}$ are nonnegative integers. Thus, each parameter $\mathcal{E}$ of multi-order $\mathbf{m}=\left(m_{1}, \ldots, m_{k}\right)$ is characterized by the conditions

$$
V_{\mathcal{E}}\left(z_{i}\right)=V_{\mathcal{E}}^{\prime}\left(z_{i}\right)=\ldots=V_{\mathcal{E}}^{\left(m_{i}-1\right)}\left(z_{i}\right)=0, \quad V_{\mathcal{E}}^{\left(m_{i}\right)}\left(z_{i}\right) \neq 0 \quad(i=1, \ldots, k) .
$$

With every multi-index $\mathbf{m}=\left(m_{1}, \ldots, m_{k}\right)$ we associate the nonnegative integer

$$
\gamma_{\mathbf{m}}:=\text { the number of indices } i(i=1, \ldots, k) \text { such that } m_{i}>0 .
$$

Theorem $2.1([9])$. Let $P$ be invertible with $\mathrm{sq}_{-} P=\kappa$, and let $\mathcal{E} \in \mathcal{S}_{0}$ be a parameter of multi-order $\mathbf{m}$. Then the meromorphic function $S=\mathbf{T}_{\Theta}[\mathcal{E}]$ belongs to the class $\mathcal{S}_{\kappa-\gamma_{\mathbf{m}}}$. The function $S$ has poles of multiplicities $m_{i}-1$ at $z_{i}\left(\right.$ if $m_{i}>1$ ) and can be extended analytically to all other interpolating points $z_{i}$. Moreover, $S\left(z_{i}\right)=F_{i}$ if $m_{i}=0$, and $S\left(z_{i}\right) \neq F_{i}$ if $m_{i}=1$.

Proof of Theorem 1.5. The proof uses ideas from the proof of a part of Theorem 1.7 in [10]. Let $F$ be a standard $\mathcal{S}_{\kappa}$ function, and let it satisfy interpolation conditions (1.3). Choose $r$ distinct points $\zeta_{1}, \ldots, \zeta_{r} \in \mathbb{D}$ and consider the Pick matrix $P_{k+r}\left(F ; z_{1}, \ldots, z_{k}, \zeta_{1}, \ldots, \zeta_{r}\right)$. Since $F$ satisfies conditions (1.3), this matrix can be written in block form as

$$
P_{k+r}\left(f ; z_{1}, \ldots, z_{k}, \zeta_{1}, \ldots, \zeta_{r}\right)=\left[\begin{array}{cc}
P & \Psi^{*} \\
\Psi & P_{r}\left(f ; \zeta_{1}, \ldots, \zeta_{r}\right)
\end{array}\right],
$$

where

$$
\Psi=\left[\begin{array}{c}
\Psi_{1} \\
\vdots \\
\Psi_{r}
\end{array}\right] \quad \text { and } \quad \Psi_{i}=\left[\begin{array}{lll}
\frac{1-F\left(\zeta_{i}\right) F\left(z_{1}\right)^{*}}{1-\zeta_{i} z_{1}^{*}} & \ldots & \frac{1-F\left(\zeta_{i}\right) F\left(z_{k}\right)^{*}}{1-\zeta_{i} z_{k}^{*}}
\end{array}\right]
$$

for $i=1, \ldots, r$. The formula for $\Psi_{i}$ can be written in terms of (1.61) as

$$
\Psi_{i}=\left[\begin{array}{ll}
1 & -F\left(\zeta_{i}\right)
\end{array}\right]\left[\begin{array}{l}
E^{*} \\
C^{*}
\end{array}\right]\left(I_{k}-\zeta_{i} T^{*}\right)^{-1} \quad(i=1, \ldots, r) .
$$

Since $F \in \mathcal{S}_{\kappa}$, the matrix $P_{k+r}$ in (2.3) has not more than $\kappa$ negative eigenvalues. Since its principal submatrix $P$ has $\kappa$ negative eigenvalues, it follows that

$$
\mathrm{sq}_{-} P_{k+r}\left(f ; z_{1}, \ldots, z_{k}, \zeta_{1}, \ldots, \zeta_{r}\right)=\kappa .
$$

Therefore the Schur complement $P_{r}\left(F ; \zeta_{1}, \ldots, \zeta_{r}\right)-\Psi P^{-1} \Psi^{*} \geq 0$ of $P$ in $P_{k+r}$ is positive semidefinite, or, more explicitly,

$$
\left[\frac{1-F\left(\zeta_{i}\right) F\left(\zeta_{j}\right)^{*}}{1-\zeta_{i} \zeta_{j}^{*}}-\Psi_{i} P^{-1} \Psi_{j}^{*}\right]_{i, j=1}^{r} \geq 0 .
$$


By (2.4) and (1.9),

$$
\begin{aligned}
& \frac{1-F\left(\zeta_{i}\right) F\left(\zeta_{j}\right)^{*}}{1-\zeta_{i} \zeta_{j}^{*}}-\Psi_{i} P^{-1} \Psi_{j}^{*} \\
& =\left[\begin{array}{ll}
1 & -F\left(\zeta_{i}\right)
\end{array}\right]\left\{\frac{J}{1-\zeta_{i} \zeta_{j}^{*}}\right. \\
& \left.-\left[\begin{array}{c}
E^{*} \\
C^{*}
\end{array}\right]\left(I-\zeta_{i} T^{*}\right)^{-1} P^{-1}\left(I-\zeta_{j}^{*} T\right)^{-1}\left[\begin{array}{ll}
E & C
\end{array}\right]\right\}\left[\begin{array}{c}
1 \\
F\left(\zeta_{j}\right)^{*}
\end{array}\right] \\
& =\left[\begin{array}{ll}
1 & -F\left(\zeta_{i}\right)
\end{array}\right] \frac{\Theta\left(\zeta_{i}\right) J \Theta\left(\zeta_{j}\right)^{*}}{1-\zeta_{i} \zeta_{j}^{*}}\left[\begin{array}{c}
1 \\
-F\left(\zeta_{j}\right)^{*}
\end{array}\right],
\end{aligned}
$$

which allows us to rewrite 2.5) as

$$
\left.\left[\begin{array}{ll}
1 & \left.-F\left(\zeta_{i}\right)\right]
\end{array}\right] \frac{\Theta\left(\zeta_{i}\right) J \Theta\left(\zeta_{j}\right)^{*}}{1-\zeta_{i} \zeta_{j}^{*}}\left[\begin{array}{c}
1 \\
-F\left(\zeta_{j}\right)^{*}
\end{array}\right]\right]_{i, j=1}^{r} \geq 0 .
$$

Making use of the block decomposition (1.8) of $\Theta$ into four scalar blocks, note that

$$
d(z):=\Theta_{21}(z) F(z)-\Theta_{11}(z) \neq 0, \quad z \notin\left\{z_{1}, \ldots, z_{k}\right\} .
$$

Indeed, assuming that $\Theta_{21}(\zeta) F(\zeta)=\Theta_{11}(\zeta)$ for some $\zeta \notin\left\{z_{1}, \ldots, z_{k}\right\}$, we get

$$
\begin{aligned}
& {\left[\begin{array}{ll}
1 & -F(\zeta)
\end{array}\right] \frac{\Theta(\zeta) J \Theta(\zeta)^{*}}{1-|\zeta|^{2}}\left[\begin{array}{c}
1 \\
-F(\zeta)^{*}
\end{array}\right]} \\
& =-\left[\begin{array}{ll}
1 & -F(\zeta)
\end{array}\right] \frac{\left[\begin{array}{l}
\Theta_{12}(\zeta) \\
\Theta_{22}(\zeta)
\end{array}\right]\left[\begin{array}{ll}
\Theta_{12}(\zeta)^{*} & \Theta_{22}(\zeta)^{*}
\end{array}\right]}{1-|\zeta|^{2}}\left[\begin{array}{c}
1 \\
-F(\zeta)^{*}
\end{array}\right] \leq 0,
\end{aligned}
$$

which contradicts (2.7), unless $\operatorname{det} \Theta(\zeta)=0$. But, as follows from (1.10), $\Theta(z)$ is invertible at each point $\zeta \notin\left\{z_{1}, \ldots, z_{k}\right\}$. Thus, the function

$$
\mathcal{E}(z)=\frac{\Theta_{12}(z)-F(z) \theta_{22}(z)}{\Theta_{21}(z) F(z)-\Theta_{11}(z)}
$$

is defined on $\mathbb{D} \backslash\left\{z_{1}, \ldots, z_{k}\right\}$. Moreover,

$$
\left[\begin{array}{ll}
1 & -F(\zeta)
\end{array}\right] \Theta(\zeta)=-d(\zeta)^{-1}\left[\begin{array}{ll}
1 & -\mathcal{E}(\zeta)
\end{array}\right],
$$

and thus inequality (2.7) can be written in terms of $\mathcal{E}$ as

$$
\left[d\left(\zeta_{i}\right)^{-1}\left[\begin{array}{ll}
1 & -\mathcal{E}\left(\zeta_{i}\right)
\end{array}\right] \frac{J}{1-\zeta_{i} \zeta_{j}^{*}}\left[\begin{array}{c}
1 \\
-\mathcal{E}\left(\zeta_{j}\right)^{*}
\end{array}\right]\left(d\left(\zeta_{j}\right)^{*}\right)^{-1}\right]_{i, j=1}^{r} \geq 0,
$$

or equivalently (since $d\left(\zeta_{i}\right) \neq 0$ ), as

$$
\left[\frac{1-\mathcal{E}\left(\zeta_{i}\right) \mathcal{E}\left(\zeta_{j}\right)^{*}}{1-\zeta_{i} \zeta_{j}^{*}}\right]_{i, j=1}^{r} \geq 0
$$

Since the integer $r$ and the points $\zeta_{1}, \ldots, \zeta_{r}$ are arbitrary, the latter inequality means that $\mathcal{E}(z)$ is a Schur function. It follows from (2.9) that

$$
F(z)=\frac{\Theta_{11}(z) \mathcal{E}(z)+\Theta_{12}(z)}{\Theta_{21}(z) \mathcal{E}(z)+\Theta_{22}(z)}
$$

at every point $z \in \mathbb{D} \backslash\left\{z_{1}, \ldots, z_{k}\right\}$. 
Conversely, let $F$ be defined via (1.13) for some $\mathcal{E} \in \mathcal{S}_{0}$. Then it satisfies the interpolation conditions (1.3), by definition. It remains to show that $F$ is a standard function from the class $\mathcal{S}_{\kappa}$. Let $\mathcal{E}$ be a parameter of multi-order $\mathbf{m}$. Then by Theorem 2.1, the function $\mathbf{T}_{\Theta}[\mathcal{E}]$ (the meromorphic part of $F$ ) belongs to the class $\mathcal{S}_{\kappa-\gamma_{\mathbf{m}}}$ and satisfies

$$
\mathbf{T}_{\Theta}[\mathcal{E}]\left(z_{i}\right)=F_{i} \quad \text { if } \quad m_{i}=0 ; \quad \mathbf{T}_{\Theta}[\mathcal{E}]\left(z_{i}\right) \neq F_{i} \quad \text { if } \quad m_{i}>0 .
$$

Thus, the function $F$ is obtained via (1.13) by adding $\gamma_{\mathbf{m}}$ jumps to the meromorphic function $\mathbf{T}_{\Theta}[\mathcal{E}]$. By [10, Theorems 1.7, 1.8], $F$ belongs to $\mathcal{S}_{\kappa}$. Clearly, $F$ is a standard function.

\section{Degenerate problem}

In this section we consider the case when the Pick matrix $P$ of the $\mathbf{N P}_{\kappa}$ problem is singular and show that it has a unique solution. Let the $\mathbf{N P}_{\kappa}$ problem be given with $n$ interpolation conditions

$$
F\left(z_{i}\right)=F_{i} \quad(i=1, \ldots, n)
$$

such that the rank of the associated Pick matrix

$$
\widehat{P}=\left[\frac{1-F_{i} F_{j}^{*}}{1-z_{i} z_{j}^{*}}\right]_{i, j=1}^{n}
$$

equals $k<n$, whereas sq- $(\widehat{P})=\kappa$. Without loss of generality, we can assume that the top $k \times k$ principal submatrix $P$ of $\widehat{P}$ is invertible. Thus,

$$
\widehat{P}=\left[\begin{array}{cc}
P & P_{1}^{*} \\
P_{1} & P_{2}
\end{array}\right], \quad \text { where } \quad P_{2}=P_{1} P^{-1} P_{1}^{*} \quad \text { and } \quad \mathrm{sq}_{-}(P)=\kappa .
$$

Since the $(i, j)$-th entry in $P_{2}$ equals $\left(1-F_{k+i} F_{k+j}^{*}\right) /\left(1-z_{k+i} z_{k+j}^{*}\right)$ and since the $q$-th row of $P_{1}$ equals

$$
\left(E^{*}-F_{k+q} C^{*}\right)\left(I_{k}-z_{q} T^{*}\right)^{-1}
$$

(this follows from (2.3) and (2.4) with $\zeta_{q}$ replaced by $z_{k+q}$ ), we conclude upon comparing the corresponding entries in the equality $P_{2}=P_{1} P^{-1} P_{1}^{*}$ that

$$
\frac{1-F_{i} F_{j}^{*}}{1-z_{i} z_{j}^{*}}=\left(E^{*}-F_{i} C^{*}\right)\left(I-z_{i} T^{*}\right)^{-1} P^{-1}\left(I-z_{j}^{*} T\right)^{-1}\left(E-C F_{j}^{*}\right)
$$

for $j, i=k+1, \ldots, n$.

Theorem 3.1. A degenerate $\mathbf{N P}_{\kappa}$ problem has a unique solution in the class of standard functions in $\mathcal{S}_{\kappa}$, and the meromorphic part of the solution is the ratio of two Blaschke products: the numerator is of degree $k$ and the degree of the denominator does not exceed $\kappa$.

Proof. First we describe the set of all functions $F \in \mathcal{S}_{\kappa}$ that satisfy the first $k$ interpolation conditions in (3.1). The Pick matrix of this reduced interpolation problem is $P$, which is not singular and has $\kappa$ negative eigenvalues. By Theorem 1.5, all functions $F \in \mathcal{S}_{\kappa}$ subject to $F\left(z_{i}\right)=F_{i}(i=1, \ldots, k)$ are parametrized by formula (1.13), where $\Theta$ is given by (1.8) and $\mathcal{E}$ is a free parameter from $\mathcal{S}_{0}$. It remains to find all parameters $\mathcal{E}$ such that the function $F$ of the form (1.13) satisfies the remaining $n-k$ interpolation conditions in (B.1). 
Note that the meromorphic part $\mathbf{T}_{\Theta}[\mathcal{E}]$ of $F$ must satisfy these conditions:

$$
z_{i} \in \operatorname{Dom}\left(\mathbf{T}_{\Theta}[\mathcal{E}]\right) \text { and } \quad \mathbf{T}_{\Theta}[\mathcal{E}]\left(z_{i}\right)=F_{i} \quad(i=k+1, \ldots, n) .
$$

Otherwise, a jump is added to $F$ and, by Theorem [1.3, the maximal number of negative eigenvalues of Pick matrices is bigger than $\kappa$, a contradiction. Now we show that every parameter $\mathcal{E}$ such that $V_{\mathcal{E}}\left(z_{i}\right)=0$ for at least one $i \in\{k+1, \ldots, n\}$, does not lead to a solution. If $V_{\mathcal{E}}$ and $U_{\mathcal{E}}$ have a common zero at $z$, it follows from (2.1) that $\Theta(z)$ is singular, which may happen only for $z \in\left\{z_{1}, \ldots, z_{k}\right\}$, in view of (1.10). Therefore, if $V_{\mathcal{E}}\left(z_{i}\right)=0$ for some $i \in\{k+1, \ldots, n\}$, then $U_{\mathcal{E}}\left(z_{i}\right) \neq 0$ and $\mathbf{T}_{\Theta}[\mathcal{E}]$ has a pole at $z_{i}$ and cannot satisfy the corresponding condition in (3.5). Thus, we are staying with parameters $\mathcal{E}$ for which $\mathbf{T}_{\Theta}[\mathcal{E}]$ is analytic at $z_{k+1}, \ldots, z_{n}$; these are exactly the parameters for which

$$
\Theta_{21}\left(z_{i}\right) \mathcal{E}\left(z_{i}\right)+\Theta_{22}\left(z_{i}\right) \neq 0, \quad \text { for } i=k+1, \ldots, n .
$$

For these parameters, conditions (3.5) are equivalent to

$$
a_{i} \mathcal{E}\left(z_{i}\right)=-c_{i} \quad(i=k+1, \ldots, n),
$$

where $a_{i}, c_{i}$ are given by

$$
\left[\begin{array}{ll}
a_{j} & c_{j}
\end{array}\right]=\left[\begin{array}{ll}
1 & -F_{j}
\end{array}\right] \Theta\left(z_{j}\right), \quad j=k+1, \ldots, n .
$$

Indeed, multiplying the $i$-th interpolation condition in (3.5) by $\Theta_{21}\left(z_{j}\right) \mathcal{E}\left(z_{i}\right)+$ $\Theta_{22}\left(z_{i}\right) \neq 0$, we get

$$
\Theta_{11}\left(z_{i}\right) \mathcal{E}\left(z_{i}\right)+\Theta_{12}\left(z_{i}\right)=F_{i}\left(\Theta_{21}\left(z_{i}\right) \mathcal{E}\left(z_{i}\right)+\Theta_{22}\left(z_{i}\right)\right)
$$

and, after clear rearrangements,

$$
\left(\Theta_{11}\left(z_{i}\right)-F_{i} \Theta_{21}\left(z_{j}\right)\right) \mathcal{E}\left(z_{i}\right)=F_{i} \Theta_{22}\left(z_{i}\right)-\Theta_{12}\left(z_{i}\right) .
$$

The latter equality can be written as

$$
\left[\begin{array}{ll}
1 & -F_{j}
\end{array}\right]\left[\begin{array}{c}
\Theta_{11}\left(z_{i}\right) \\
\Theta_{21}\left(z_{i}\right)
\end{array}\right] \mathcal{E}\left(z_{i}\right)=-\left[\begin{array}{ll}
1 & -F_{j}
\end{array}\right]\left[\begin{array}{c}
\Theta_{12}\left(z_{i}\right) \\
\Theta_{22}\left(z_{i}\right)
\end{array}\right],
$$

which coincides with (3.7), on account of (3.6).

Making use of (3.4), along with (3.8) and (1.9), we get (for $j, i=k+1, \ldots, n)$

$$
\begin{aligned}
& \frac{a_{i} a_{j}^{*}-c_{i} c_{j}^{*}}{1-z_{i} z_{j}^{*}} \\
= & {\left[\begin{array}{ll}
a_{i} & c_{i}
\end{array}\right] \frac{J}{1-z_{i} z_{j}^{*}}\left[\begin{array}{c}
a_{j}^{*} \\
c_{j}^{*}
\end{array}\right]=\left[\begin{array}{ll}
1 & -F_{i}
\end{array}\right] \frac{\Theta\left(z_{i}\right) J \Theta\left(z_{j}\right)^{*}}{1-z_{i} z_{j}^{*}}\left[\begin{array}{c}
1 \\
-F_{j}^{*}
\end{array}\right] } \\
= & {\left[\begin{array}{ll}
1 & -F_{i}
\end{array}\right] \frac{J}{1-z_{i} z_{j}^{*}}\left[\begin{array}{c}
1 \\
-F_{j}^{*}
\end{array}\right] } \\
& -\left[\begin{array}{ll}
1 & -F_{i}
\end{array}\right]\left[\begin{array}{c}
E^{*} \\
C^{*}
\end{array}\right]\left(I-z T^{*}\right)^{-1} P^{-1}\left(I-w^{*} T\right)^{-1}\left[\begin{array}{ll}
E & C
\end{array}\right]\left[\begin{array}{c}
1 \\
-F_{j}^{*}
\end{array}\right] \\
= & \frac{1-F_{i} F_{j}^{*}}{1-z_{i} z_{j}^{*}}-\left(E^{*}-F_{i} C^{*}\right)\left(I-z_{i} T^{*}\right)^{-1} P^{-1}\left(I-z_{j}^{*} T\right)^{-1}\left(E-C F_{j}^{*}\right)=0 .
\end{aligned}
$$

Thus, the Pick matrix of the interpolation problem (3.7) is the zero matrix. Note also that $a_{j}=0$ for $j=k+1, \ldots, n$; otherwise, in view of $\left|a_{j}\right|=\left|c_{j}\right|$ $(j=k+1, \ldots, n)$, we would have $a_{j}=c_{j}=0$ for some $j=k+1, \ldots, n$. Hence (by $(\underline{3.8})) \Theta\left(z_{j}\right)$ is singular, a contradiction with (1.10). 
Therefore, there is only one $\mathcal{E} \in \mathcal{S}_{0}$ satisfying conditions (3.7), and this $\mathcal{E}$ is a unimodular constant:

$$
\mathcal{E}=-\frac{c_{k+1}}{a_{k+1}}=\ldots=-\frac{c_{n}}{a_{n}}
$$

Next, we verify that (3.6) holds for $\mathcal{E}$ given by (3.9). We have, for a fixed $j$ $(j=k+1, \ldots, n)$,

$$
\begin{aligned}
\Theta_{21}\left(z_{j}\right) \mathcal{E}+\Theta_{22}\left(z_{j}\right) & =\Theta_{21}\left(z_{j}\right)-\Theta_{22}\left(z_{j}\right) \frac{c_{j}}{a_{j}}=\frac{1}{a_{j}}\left[\begin{array}{ll}
a_{j} & c_{j}
\end{array}\right]\left[\begin{array}{c}
\Theta_{22}\left(z_{j}\right) \\
-\Theta_{21}\left(z_{j}\right)
\end{array}\right] \\
(\text { by (3.8) }) & =\frac{\operatorname{det} \Theta\left(z_{j}\right)}{a_{j}}\left[\begin{array}{ll}
1 & -F_{j}
\end{array}\right] \Theta\left(z_{j}\right)\left(\Theta\left(z_{j}\right)\right)^{-1}\left[\begin{array}{c}
1 \\
0
\end{array}\right]=1 .
\end{aligned}
$$

It remains to note that the function $\mathbf{T}_{\Theta}[\mathcal{E}]$, with $\mathcal{E}$ as in (3.9), is the ratio of Blaschke products of desired degrees.

In [11] it was shown that the degenerate $\mathbf{N P}_{\kappa}$ problem does not always have a solution in the class of meromorphic functions, under the hypothesis that (1.4) has $\kappa$ negative eigenvalues. In contrast, as follows from Theorems [1.5] and 3.1] in the class $\mathcal{S}_{\kappa}$ a solution always exists, under the same hypothesis.

\section{An extension RESUlt}

Theorem 4.1. Let $F$ be a function defined on a set $\Omega \subseteq \mathbb{D}$ and such that the Pick matrices $P_{n}\left(F ; z_{1}, \ldots, z_{n}\right), z_{1}, \ldots, z_{n} \in \Omega$, have at most $\kappa$ negative eigenvalues (counted with multiplicities) and at least one of them has exactly $\kappa$ negative eigenvalues. Then $F$ admits an extension to a standard function in the class $\mathcal{S}_{\kappa}$.

Proof. If $\Omega$ is a set of uniqueness of $H^{\infty}$, then Theorem 4.1 follows from [4, Theorem 2.1] coupled with Theorem 1.3 If $\Omega$ is finite, then Theorem 4.1 follows from Theorems 1.4 and 3.1. Thus, it remains to consider the case when $\Omega$ is discrete (and infinite). Let $\Omega=\left\{z_{1}, \ldots, z_{n}, \ldots\right\}$. If for some $n_{0}$ the Pick matrix $P_{n_{0}}\left(F ; z_{1}, \ldots, z_{n_{0}}\right)$ has $\kappa$ negative eigenvalues and is singular, then by Theorem 3.1 there exists a unique standard function $\tilde{F} \in \mathcal{S}_{\kappa}$ such that $\tilde{F}\left(z_{j}\right)=F\left(z_{j}\right), j=1, \ldots, n_{0}$. Then $\tilde{F}\left(z_{j}\right)=F\left(z_{j}\right)$ for $j=n_{0}+1, \ldots$ (see the proof of Theorem 3.1). We are left with the case when the Pick matrices $P_{n}\left(F ; z_{1}, \ldots, z_{n}\right)$ that have $\kappa$ negative eigenvalues are all nonsingular.

Let $f_{n}$ be a meromorphic solution to the Nevanlinna-Pick interpolation problem with Pick matrix $P_{n}\left(F ; z_{1}, \ldots, z_{n}\right)$. Then (see the Krein-Langer formula 14]) $f_{n}=$ $s_{n} / b_{n}$, where $s_{n}$ is a Schur function and $b_{n}$ is a Blaschke product of degree $\kappa$ normalized so that $b_{n}(1)=1$. By Montel's theorem (see, e.g., 15]), there exists a Schur function $s$ and a subsequence $n_{k}$ so that $\lim _{k \rightarrow \infty} s_{n_{k}}(z)=s(z)$ uniformly on compact subsets of $\mathbb{D}$. By passing to a subsequence of $n_{k}$ we can also ensure that $\lim _{k \rightarrow \infty} b_{n_{k}}(z)=b(z)$ uniformly on compact subsets of $\mathbb{D}$. The limit function $b(z)$ is a Blaschke product of degree $\kappa^{\prime} \leq \kappa$. In fact, if we enumerate the zeros $\left\{\mu_{n_{k}}^{(j)}\right\}_{j=1}^{\kappa}$ of $b_{n_{k}}(z)$, then (again by passing to a subsequence of $n_{k}$ ) we can make $\lim _{k \rightarrow \infty} \mu_{n_{k}}^{(i)}=\mu^{(i)}$. If $\left|\mu^{(i)}\right|<1$, the Blaschke factors with zeros at $\mu_{n_{k}}^{(i)}$ converge to the Blaschke factor with zero at $\mu^{(i)}$. If $\left|\mu^{(i)}\right|=1$, then they converge to a unimodular constant. 
We now define the function

$$
f(z)=\frac{s(z)}{b(z)} \text { if } b(z) \neq 0, \quad f\left(z_{i}\right)=F_{i} \text { if } z=z_{i} \in \Omega .
$$

Note first that the definition is correct. Indeed, if $b\left(z_{i}\right) \neq 0, z_{i} \in \Omega$, then

$$
\frac{s\left(z_{i}\right)}{b\left(z_{i}\right)}=\frac{\lim _{k \rightarrow \infty} s_{n_{k}}\left(z_{i}\right)}{\lim _{k \rightarrow \infty} b_{n_{k}}\left(z_{i}\right)}=\lim _{k \rightarrow \infty} \frac{s_{n_{k}}\left(z_{i}\right)}{b_{n_{k}}\left(z_{i}\right)}=\lim _{k \rightarrow \infty} f_{n_{k}}\left(z_{i}\right)=\lim _{k \rightarrow \infty} F_{i}=F_{i} .
$$

The domain of definition of $f$ is $\mathbb{D} \backslash\{z \in \mathbb{D}: b(z)=0, z \notin \Omega\}$. Observe next that

$$
f(z)=\lim _{k \rightarrow \infty} f_{n_{k}}(z), \quad z \in \operatorname{Dom}(f) .
$$

Indeed, if $b(z) \neq 0$, then

$$
f(z)=\frac{s(z)}{b(z)}=\frac{\lim _{k \rightarrow \infty} s_{n_{k}}(z)}{\lim _{k \rightarrow \infty} b_{n_{k}}(z)}=\lim _{k \rightarrow \infty} \frac{s_{n_{k}}(z)}{b_{n_{k}}(z)}=\lim _{k \rightarrow \infty} f_{n_{k}}(z),
$$

and if $z_{i} \in \Omega$, then $f_{n_{k}}\left(z_{i}\right)=F_{i}$ for all sufficiently large $k$. Thus by definition, $f$ solves the Nevanlinna-Pick interpolation problem on the set $\Omega$. Since the Pick matrices of $f$ are limits of the corresponding Pick matrices of $f_{n_{k}}$, they have at most $\kappa$ negative eigenvalues. Since some Pick matrices of the restriction of $f$ to $\Omega$ already have $\kappa$ negative eigenvalues, it follows that $f \in S_{\kappa}$.

We conclude with an example showing a situation when a function $F$, defined on a discrete set $\Omega \subset \mathbb{D}$ such that the maximal number of negative eigenvalues of its Pick matrices is equal to one, admits a unique extension to a standard function in class $\mathcal{S}_{1}$, and this unique extension is not meromorphic in $\mathbb{D}$. At the same time, all restrictions of $F$ to finite subsets of $\Omega$ give rise to nondegenerate $\mathbf{N P}_{1}$ problems which therefore have infinitely many meromorphic solutions. Let $\Omega=\{0\} \cup\left\{z_{k}\right\}_{k=1}^{\infty}$, where $z_{k} \in \mathbb{D}$ are distinct nonzero numbers such that $\sum_{k=1}^{\infty}\left(1-\left|z_{k}\right|\right)^{2}<\infty$. Let $\widehat{w}$ be a non-inner Schur function which is a unique Schur function that solves the interpolation problem $w\left(z_{k}\right)=\widehat{w}\left(z_{k}\right)$, for $k=1,2, \ldots$ The existence of such a $\widehat{w}$ was demonstrated in [2] and [3]. Consider two matrix functions

$$
\Theta_{1 / 2}(z)=\frac{1}{3}\left[\begin{array}{cc}
4 z-1 & 2-2 z \\
2 z-2 & 4-z
\end{array}\right], \quad \Theta_{2}(z)=\frac{1}{3}\left[\begin{array}{cc}
4-z & 2 z-2 \\
2-2 z & 4 z-1
\end{array}\right],
$$

which are specifications of (1.8) to the case $k=1, T=0, E=1, C=1 / 2$, and to the case $k=1, T=0, E=1, C=2$, respectively. The formula

$$
w=\mathbf{T}_{\Theta_{1 / 2}}[\mathcal{E}], \quad \mathcal{E} \in \mathcal{S}_{0},
$$

parametrizes the set of solutions $w \in \mathcal{S}_{0}$ of the Nevanlinna-Pick interpolation problem $w(0)=1 / 2($ see Theorem 1.4 $)$. Define

$$
w_{1 / 2}=\mathbf{T}_{\Theta_{1 / 2}}[\widehat{w}] .
$$

Since $\widehat{w}$ is not an inner function, $w_{1 / 2}$ is not an inner function either.

Proposition 4.2. Each of the two Nevanlinna-Pick problems

$$
w(0)=1 / 2, \quad w\left(z_{k}\right)=w_{1 / 2}\left(z_{k}\right), \quad k=1,2, \ldots, \quad w \in \mathcal{S}_{0},
$$

and

$$
w\left(z_{k}\right)=w_{1 / 2}\left(z_{k}\right), \quad k=1,2, \ldots, \quad w \in \mathcal{S}_{0},
$$

has a unique solution $w$, and $w=w_{1 / 2}$. 
Proof. Assume that $w$ solves the problem (4.3). Since $w(0)=1 / 2$, there exists $\mathcal{E} \in \mathcal{S}_{0}$ such that

$$
w=\mathbf{T}_{\Theta_{1 / 2}}[\mathcal{E}] .
$$

One verifies (using (4.2) and (4.5) that $\mathcal{E}\left(z_{k}\right)=\widehat{w}\left(z_{k}\right)$ for $k=1,2, \ldots$, and therefore $\mathcal{E}=\widehat{w}$ by our choice of $\widehat{w}$.

Next, assume that the solution of (4.4) is not unique. It is well known [16, [18 that in this case the solution set of (4.4) is described by the formula $w=\mathbf{T}_{\Theta}[\mathcal{E}]$, where $\Theta$ is a fixed $J$-inner $2 \times 2$ matrix function, and $\mathcal{E} \in \mathcal{S}_{0}$ is a free parameter. Since $w_{1 / 2}$ solves (4.4), we have $w_{1 / 2}=\mathbf{T}_{\Theta}\left[\mathcal{E}_{1 / 2}\right]$ for some $\mathcal{E}_{1 / 2} \in \mathcal{S}_{0}$. Observe that if $\mathcal{E}(0)=\mathcal{E}_{1 / 2}(0), \mathcal{E} \in \mathcal{S}_{0}$, then $\mathbf{T}_{\Theta}[\mathcal{E}](0)=1 / 2$. Thus, if it were $\left|\mathcal{E}_{1 / 2}(0)\right|<1$, then there would exist infinitely many functions $\mathcal{E} \in \mathcal{S}_{0}$ such that $\mathcal{E}(0)=\mathcal{E}_{1 / 2}(0)$, and therefore (4.3) would have infinitely many solutions, a contradiction with the already proved part of Proposition 4.2. Also, if it were $\left|\mathcal{E}_{1 / 2}(0)\right|=1$, then $\mathcal{E}_{1 / 2}$ would be a unimodular constant, and hence $w_{1 / 2}$ would be an inner function, a contradiction again.

By Theorem 1.5 the standard solutions of the $\mathbf{N P}_{1}$ problem $w(0)=2$ are given by the formula

$$
w(z)=\mathbf{G}_{\Theta_{2}}[\mathcal{E}]:=\left\{\begin{array}{cl}
\mathbf{T}_{\Theta_{2}}[\mathcal{E}] & \text { if } z \neq 0, \\
2 & \text { if } z=0,
\end{array} \quad \mathcal{E} \in \mathcal{S}_{0} .\right.
$$

Let $w_{0}=\mathbf{G}_{\Theta_{2}}\left[w_{1 / 2}\right]$. Note that $w_{0}$ has a jump but not a pole (since both the denominator and the numerator of $\mathbf{T}_{\Theta_{2}}\left[w_{1 / 2}\right]$ vanish at zero) at the origin. Consider the interpolation problem

$$
w(0)=2, \quad w\left(z_{k}\right)=w_{0}\left(z_{k}\right), \quad k=1,2, \ldots, \quad w \in \mathcal{S}_{1} .
$$

The function $w_{0}$ is the unique standard solution of (4.6). Indeed, if $w$ is a standard solution of (4.6), then $w=\mathbf{G}_{\Theta_{2}}[\mathcal{E}]$ for some $\mathcal{E} \in \mathcal{S}_{0}$. Since $w\left(z_{k}\right)=w_{0}\left(z_{k}\right)=$ $\mathbf{T}_{\Theta_{2}}\left[w_{1 / 2}\right]\left(z_{k}\right)$, the function $\mathcal{E}$ satisfies $\mathcal{E}\left(z_{k}\right)=w_{1 / 2}\left(z_{k}\right)$ for $k=1,2, \ldots$, and therefore by Proposition 4.2 we must have $\mathcal{E}=w_{1 / 2}$.

\section{REFERENCES}

1. V. M. Adamjan, D. Z. Arov, and M. G. Krel̆n, Analytic properties of the Schmidt pairs of a Hankel operator and the generalized Schur-Takagi problem, (Russian) Mat. Sb. (N.S.) 86(128) (1971), 34-75. MR 45:7505

2. V. M. Adamjan, D. Z. Arov, and M. G. Krein, Bounded operators which commute with a $C_{00}$ class contraction whose rank of nonunitarity is one, (Russian) Funkcional Anal. i Prilozhen 3 (1969), 86-87. MR 41:5990

3. V. M. Adamjan, D. Z. Arov, and M. G. Krein, Infinite Hankel matrices and generalized problems of Carathéodory-Fejér and F. Riesz, (Russian) Funkcional Anal. i Prilozhen 2 (1968), 1-19. MR 38:2591

4. J. Agler and N. J. Young, Functions which are almost multipliers of Hilbert function spaces, Proc. London Math. Soc. 76 (1998), 453-475. MR 99g:46027

5. D. Alpay, A. Dijksma, J. Rovnyak, and H. de Snoo, Schur functions, operator colligations and reproducing kernel Pontryagin spaces, Operator Theory: Advances and Applications, vol. 96, Birkhäuser-Verlag, Basel, 1997. MR 2000a:47024

6. J. A. Ball, I. Gohberg, and L. Rodman, Interpolation of rational matrix functions, Operator Theory: Advances and Applications, vol. 45, Birkhäuser-Verlag, Basel, 1990. MR 92m:47027

7. J. A. Ball and J. W. Helton, A Beurling-Lax theorem for the Lie group $\mathbf{U}(m, n)$ which contains most classical interpolation theory, J. Operator Theory 9 (1983), 107-142. MR 84m:47046 
8. J. A. Ball and J. W. Helton, Interpolation problems of Pick-Nevanlinna and Loewner types for meromorphic matrix functions: parametrization of the set of all solutions, Integral Equations and Operator Theory 9 (1986), 155-203. MR 87j:30085

9. V. Bolotnikov, On the Carathéodory-Fejér interpolation for generalized Schur functions, preprint.

10. V. Bolotnikov, A. Kheifets, and L. Rodman, Functions with Pick matrices having bounded number of negative eigenvalues, Contemporary Mathematics 323 (2003), 393-417.

11. T. Constantinescu and A. Gheondea, The Schur algorithm and coefficient characterizations for generalized Schur functions, Proc. Amer. Math. Soc. 128 (2000), no. 9, 2705-2713. MR 2000m:47015

12. A. Dijksma and H. Langer, Notes on a Nevanlinna-Pick interpolation problem for generalized Nevanlinna functions, in: Topics in interpolation theory, Oper. Theory Adv. Appl., 95 (1997), 69-91. MR 98g:47015

13. L. B. Golinskii, A generalization of the matrix Nevanlinna-Pick problem, Izv. Akad. Nauk Armyan. SSR Ser. Mat. 18 (1983), 187-205. (Russian). MR 85g:47049

14. M. G. KreĬn and H. Langer, Über die verallgemeinerten Resolventen und die charakteristische Funktion eines isometrischen Operators im Raume $\Pi_{\kappa}$, Colloq. Math. Soc. János Bolyai 5, North-Holland, Amsterdam, 1972, pp. 353-399. MR 54:11103

15. J. E. Marsden and M. J. Hoffman, Basic complex analysis, second ed., W. H. Freeman and Company, NY, 1987. MR 88m:30001

16. R. Nevanlinna, Über beschränkte analytische Funktionen, Ann. Acad. Sci. Fenn. Ser. A 32 (1929), no. 7 .

17. A. A. Nudelman, A generalization of classical interpolation problems, Dokl. Akad. Nauk. SSSR 256 (1981), 790-793. (Russian). MR 82f:30033

18. J. L. Walsh, Interpolation and approximation by rational functions in the complex domain, fourth ed., Amer. Math. Soc., Providence, R.I., 1960. MR 36:1672a

Department of Mathematics, The College of William and Mary, Williamsburg, VirGINIA 23187-8795

E-mail address: vladi@math.wm.edu

Department of Mathematics, The College of William and Mary, Williamsburg, VirGINIA 23187-8795

E-mail address: sykhei@wm.edu

Department of Mathematics, The College of William and Mary, Williamsburg, VirGINIA 23187-8795

E-mail address: lxrodm@math.wm.edu 